\title{
ENSAMBLE FICOPERIFÍTICO ASOCIADO A MACRÓFITAS EN UNA CIÉNAGA TROPICAL COLOMBIANA
}

\author{
PHYCOPERIPHYTON ASSEMBLAGE ASSOCIATED TO MACROPHYTES IN A TROPICAL \\ COLOMBIAN SWAMP
}

\author{
Luis Alberto De La Hoz-Barrientos y Farid Jesús Osorio-Ávila
}

\begin{abstract}
RESUMEN
El ficoperifíton cumple un papel fundamental en la dinámica de los humedales, pues posee atributos importantes para la bioindicación ya que es sensible a modificaciones del medio en el que habita. El propósito del presente trabajo es determinar la composición y estructura del ensamble ficoperifítico asociado a las raíces de macrófitas en una ciénaga tropical del noroccidente de Colombia. Se realizaron tres muestreos (junio de 2015, setiembre de 2015 y enero de 2016) en tres estaciones localizadas en la zona oriental de la ciénaga. En cada estación se recolectaron muestras de $5 \mathrm{~cm}^{2}$ de raíces de tres individuos de cada macrófita dominante, simultáneamente, se midieron in situ variables físicas y químicas del agua (pH, conductividad, oxígeno, temperatura, profundidad, extinción de luz, sólidos sedimentables y radiación). Se reportan 81 morfoespecies, siendo las diatomeas y clorófitas las algas dominantes. La macrófita Eichhornia crassipes fue el sustrato con mayor riqueza y densidad algal. Cymbella, Gomphonema, Monoraphidium y Cosmarium fueron considerados como colonizadores exitosos, encontrándose en la mayoría de los sustratos evaluados. Las algas perifíticas por su alta tasa de reciclaje de nutrientes han de desempeñar un papel fundamental en la dinámica de este humedal.
\end{abstract}

PALABRAS CLAVE: Perifíton, macrófitas, humedales, ensamble.

\begin{abstract}
The phycoperiphyton plays a fundamental role in the dynamic of wetlands due to the fact that possess important attributes as bio-indicators and because of its sensitivity to environmental changes. The aim of this paper is to determine the composition and structure of phycoperiphyton assemblage related to macrophytes' roots at a tropical swamp in the northwest of Colombian. Three samples were taken (June 2015, September 2015 and January 2016) in three separate locations in the eastern area of the swamp. In each location a sample of $5 \mathrm{~cm}^{2}$ was taken from the roots of three individuals for each dominant macrophyte. Simultaneously, in situ water chemical and physical variables were measured ( $\mathrm{pH}$, conductivity, oxygen, temperature, depth, light extinction, sedimentary solids and radiation). Reports of 81 morphospecies were found, being diatoms and chlorophytes the dominant algae. The macrophyte Eichornia crassipes were the richest substratum with the highest algae density. Cymbella, Gomphonema, Monoraphidium y Cosmarium were considered as successful colonizers, as they were found in the majority of the substratum evaluated. Due to its high nutrient recycling rate, periphytic algae plays a fundamental role in the dynamics of this swamp.
\end{abstract}

KEY WORDS: Periphyton, macrophytes, wetlands, assemblage.

La definición de ensamble ha sido un tema de mucha controversia, generando diferentes terminologías, algunas más o menos aceptadas, de la cuales muchas son consideradas sinónimos del termino comunidad (Stroud et al., 2015). El ensamble es una fracción de la comunidad seleccionada desde el punto de vista taxonómico (Ramírez y Gutiérrez-Fonseca, 2016) ya que se encuentra constituido por un grupo de especies pertenecientes a un taxón y que comparten los mismos recursos en un espacio geográfico (Fauth et al., 1996). Se considera perifíton al agregado de microorganismos (algas, hongos, bacterias y protozoos) asociados a 
sustratos sumergidos, los cuales pueden ser naturales o artificiales (Wetzel, 2001). Dentro de este componente, el ensamble algal desempeña un rol fundamental en la dinámica de los humedales, en los que se destaca su aporte a la productividad primaria y la alta tasa de reciclaje de nutrientes, considerándose indicadores de la calidad del agua ya que reflejan los cambios ocurridos por alteraciones físicas, químicas y biológicas en el ecosistema (Sand-Jensen, 1983; Vadeboncoeur y Steinman, 2002; Goldsborough et al., 2005).

En Colombia, existe información insuficiente respecto a los ensambles ficoperifíticos, especialmente en ciénagas (Montoya-Moreno y Aguirre, 2009), pues los estudios se han enfocado principalmente en ecosistemas como embalses (Sierra y Ramírez, 2000), lagos (Donato et al., 1996) y ríos (Hernández-Atilano et al., 2005; Montoya-Moreno y Ramírez, 2007). En el departamento del Magdalena, Osorio y Manjarres (2015) encontraron en la ciénaga Cerro de San Antonio que el ensamble ficoperifítico presentó baja variación en cuanto a los índices comunitarios (riqueza, diversidad y dominancia) y no se encontraron diferencias en la estructura del ensamble algal entre las raíces de las diferentes macrófitas. Sin embargo, si hubo diferencia en la abundancia del ficoperifiton por unidad de área en las diferentes raíces de las macrófitas estudiadas.

En la ciénaga de Chilloa no hay estudios publicados que establezcan la estructura y la composición del ensamble algal, por lo cual, generar información de línea base sobre la ecología de estos ensambles puede servir de insumo para futuras investigaciones que permitan la evaluación ambiental en este ecosistema. Teniendo en cuenta la importancia ecológica de la asociación perifíton-macrófitas para el funcionamiento de los ecosistemas acuáticos poco profundos (Kiss et al., 2003), el objetivo principal de este estudio fue determinar la composición y la estructura del ensamble ficoperifítico en la ciénaga de Chilloa, Magdalena.

La ciénaga de Chilloa se ubica sobre el denominado ecosistema de humedales del sur, a una altitud de 17 msnm, al noroccidente de Colombia $\left(09^{\circ} 04^{\prime} \mathrm{N}, 73^{\circ} 58^{\prime}\right.$ W y $09^{\circ} 12^{\prime} \mathrm{N}, 74^{\circ} 10^{\prime} \mathrm{W}$ ) (FUPARCIS, 2014).

Se seleccionaron tres estaciones de muestreo en la zona oriental de la ciénaga, a partir de visitas previas en las que se definieron los siguientes criterios de selección: (1) Presencia de macrófitas acuáticas de diferentes especies y (2) Accesibilidad a la zona (Figura 1).

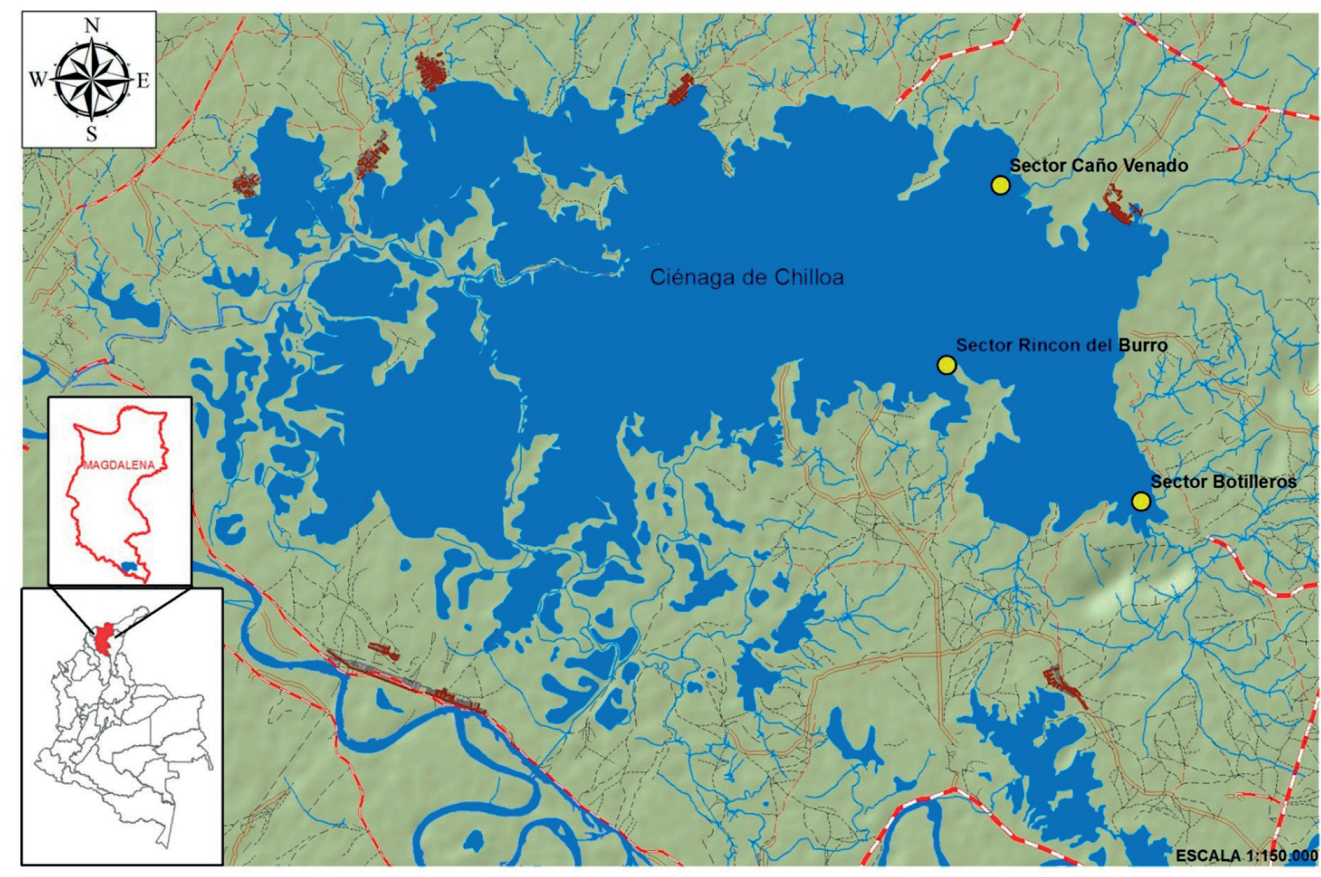

Figura 1. Mapa de la ciénaga de Chilloa. Estaciones de muestreo: Rincón del Burro (E1), Caño Venado (E2), Botilleros (E3). Elaboró grupo de SIG FUPARCIS. 
En cada estación, se tomaron muestras de las especies de macrófitas presentes. Para la identificación de las macrófitas se siguió a Velásquez (1994) y las macrófitas identificadas en el sitio de muestreo corresponden a las especies Eichhornia crassipes (Martius), Najas arguta (Kunth), Salvinia auriculata (Aublet), Pistia stratiotes (Linneaus), Marsilea sp., Mimosa sp. y Paspalum repens (Elliot).

Se realizaron tres muestreos: junio de 2015, setiembre de 2015 y enero de 2016. La composición específica de las macrófitas fue diferente en cada uno de los meses de muestreo, estando ausentes en Caño Venado durante el muestreo de septiembre de 2015 y en Botilleros durante los muestreos de junio de 2015 y enero de 2016. Se recolectaron muestras de $5 \mathrm{~cm}^{2}$ de raíces de tres individuos de cada macrófita dominante (metodología modificada de Montoya-Moreno y Aguirre, 2008). Con ayuda de tijeras podadoras las raíces o partes de la planta fueron cortadas y depositadas en frascos de plástico de $50 \mathrm{~mL}$ con solución Transeau (Bicudo y Menezes, 2006). De forma simultánea, se midieron in situ variables físicas y químicas del agua, tales como $\mathrm{pH}$, conductividad, oxígeno y temperatura (sonda multiparámetros YSI 500); profundidad, extinción de luz (disco Secchi), sólidos sedimentables (conos imhoff) y radiación (luxómetro).

En el laboratorio con ayuda de un cepillo dental se rasparon las muestras de raíces para separar el material perifítico de los restos vegetales. Mediante el método de oxidación en peróxido de hidrogeno, se eliminó el exceso de materia orgánica que presentaron algunas muestras tomadas (CEN, 2003). El material fue identificado en microscopia óptica (Nikon Eclipse 200) con cámara integrada, se utilizó un objetivo ocular de 100 X. Para la identificación de las especies ficoperifíticas, se emplearon las claves de Bourrelly (1970; 1972 y 1981) para géneros de microalgas, Komárek y Anagnostidis (1999; 2005) para Cyanophyceae, y Lange-Bertalot (2001), Krammer y Lange-Bertalot (1986; 1988; 1991) para Bacillariophyceae. Además, se utilizaron bases de datos digitales (Guiry, 2015).

Para el análisis cuantitativo, las muestras se concentraron por sifoneo hasta un volumen de $10 \mathrm{~mL}$. En cámaras de conteo Sedgwick-Rafter de $1 \mathrm{~mL}$, se contaron campos aleatorios hasta 100 células del taxón más abundante a través de un microscopio (Nikon Eclipse 200) (Ramírez, 2000). Por medio de la fórmula propuesta por Hauer y Lamberti (2007), se obtuvo la cuantificación de los individuos, donde la densidad se expresa en número de individuos por área de superficie $\left(\mathrm{cm}^{2}\right)$.

La temperatura para todas las estaciones oscilo entre 30,9 y $32,9^{\circ} \mathrm{C}$, el pH fue ligeramente básico $(7,9-9,0$ unidades de $\mathrm{pH}$ ), los valores de transparencia (disco secchi) oscilaron entre $15-63 \mathrm{~cm}$ y la conductividad presento valores entre 71,4 a $179 \mu \mathrm{S} / \mathrm{cm}$. Se registraron valores de oxígeno disuelto entre 3,26 y 7,73 mg/L, luz entre 792 y 996 lux y valores de sólidos sedimentables entre 0,1 y $0,8 \mathrm{mg} / \mathrm{L}$ (Tabla 1 ).

Tabla 1. Valores de las variables físicas y químicas registradas en cada estación. Temp $=$ Temperatura agua $\left({ }^{\circ} \mathrm{C}\right)$, OD $(\%)=$ Oxígeno disuelto en porcentaje, $\mathrm{OD}=$ Oxígeno disuelto $(\mathrm{mg} / \mathrm{L})$, Cond = Conductividad $(\mu \mathrm{S} / \mathrm{cm}), \mathrm{SS}=$ Sólidos sedimentables $(\mathrm{mg} / \mathrm{L}), \mathrm{pH}(\mathrm{Unidades}$ de $\mathrm{pH})$, Transp = Transparencia $(\mathrm{cm})$, Prof = Profundidad $(\mathrm{cm})$, Rad = Radiación (lux). E1 = Rincón del Burro, E2 = Caño Venado, E3 = Botilleros. M1 = junio de 2015, M2 = septiembre de 2015, M3 = enero de 2016.

\begin{tabular}{|c|c|c|c|c|c|c|c|c|c|c|}
\hline Estación & M & Temp. & OD (\%) & OD & Cond. & SS & $\mathrm{pH}$ & Transp. & Prof. & Rad. \\
\hline \multirow{3}{*}{ E1 } & M1 & 32,9 & 90,7 & 6,58 & 143 & 0,1 & 9,0 & 63 & 170 & 987 \\
\hline & M2 & 32,5 & 80,3 & 7,09 & 134 & 0,2 & 8,6 & 42 & 128 & 989 \\
\hline & M3 & 32,3 & 65,8 & 7,61 & 71,4 & 0,4 & 8,8 & 22 & 86 & 991 \\
\hline \multirow{3}{*}{ E2 } & M1 & 30,9 & 44,6 & 3,26 & 162 & 0,8 & 8,4 & 31 & 66,5 & 792 \\
\hline & M2 & 31,8 & 57,3 & 5,48 & 153,6 & 0,7 & 8,1 & 23 & 78,2 & 886 \\
\hline & M3 & 32,7 & 70,1 & 7,73 & 145,2 & 0,7 & 7,9 & 15 & 90 & 980 \\
\hline \multirow{3}{*}{ E3 } & M1 & 31,9 & 67,6 & 4,92 & 152,5 & 0,4 & 8,7 & 18 & 81 & 989 \\
\hline & M2 & 32,1 & 88,6 & 6,31 & 179 & 2,5 & 8,3 & 22 & 84 & 996 \\
\hline & M3 & 32,5 & 67,9 & 7,67 & 148,3 & 0,5 & 8,6 & 25 & 88 & 985 \\
\hline
\end{tabular}


Debido a que los muestreos se realizaron durante un periodo de tiempo afectado por el evento "El Niño" las altas temperaturas fueron una constante. Según Algarte (2014), a mayor temperatura las reacciones enzimáticas son más rápidas, por lo tanto, el crecimiento celular aumenta. En el presente estudio, las estaciones mantuvieron un pH que permitió el crecimiento de las algas perifíticas, pues en un $\mathrm{pH}$ básico se ha observado mejor desarrollo de las comunidades ficoperifíticas, ya que bajo estas condiciones existe una mayor disponibilidad de nutrientes (Díaz-Quirós y Rivera Rondón, 2004).

La luz es considerada como uno de los factores importantes para el crecimiento de las algas perifíticas. Las clorófitas necesitan mayor intensidad de luz que las diatomeas y las cianofitas (Algarte, 2014), lo cual se vio reflejado en la estación de Botilleros donde se observó la mayor densidad de clorófitas relacionada a los mayores valores de radiación (Tabla 1). Las algas perifíticas fueron extraídas de raíces de macrófitas flotantes, estos sustratos se encuentran entre los dos y diez $\mathrm{cm}$ de profundidad, en esta zona se presenta mayor absorción lumínica (Montoya-Moreno y Aguirre, 2013a). A pesar de que existe competencia entre las macrófitas y las algas por el recurso, en los ecosistemas tropicales la intensidad lumínica es permanente durante todo el año por lo que la luz no significaría un factor limitante.

Las comunidades ficoperifíticas son alteradas por múltiples condiciones del medio en el que habitan y de la variabilidad climática. Por tanto, es difícil saber el efecto que produce cada una de las variables ambientales sobre la abundancia y la composición de las algas perifíticas, aunque la influencia de estos factores ambientales suele deducirse de la reducción de número de individuos y de cambios de las especies presentes (Díaz-Quirós y Rivera Rondón, 2004).

En Rincón del Burro (E1) se contaron 1293 células, correspondientes a 5 clases, 21 familias y 27 géneros, de las cuales el $39 \%$ (507 células / $\mathrm{cm}^{2}$ ), fueron de la clase Bacillariophyceae, seguido por Chlorophyceae con el $27 \%$ (349 células $/ \mathrm{cm}^{2}$ ). De igual forma la clase Conjugatophyceae registró una densidad de 250 células / $\mathrm{cm}^{2}$ equivalente al $19 \%$, Cyanophyceae 178 células / $\mathrm{cm}^{2}$ (14\%) y Euglenophyceae 9 células / $\mathrm{cm}^{2}(0,7 \%)$. La densidad de las algas perifíticas en las macrófitas evaluadas fue baja, debido a la alta carga de material orgánico y sedimento (observada a simple vista), el cual dificulta la fijación de las algas perifíticas en los diferentes tipos de sustratos. Condición también reportada por Montoya-Moreno y Aguirre (2008) en el complejo cenagoso de Ayapel (noroccidente de Colombia), quienes consideraron que la baja colonización algal se debía a que la biopelicula estaba principalmente conformada por material orgánico e inorgánico.

Los géneros que se encontraron con mayor abundancia en las macrófitas evaluadas en Rincón del Burro, fueron Microcystis, Dunaliella, Monoraphidium y Eunotia, siendo las dos primeras los más abundantes para $S$. auriculata (en enero de 2016) y las dos últimas para $E$. crassipes (en junio y septiembre de 2015) (Figura 2). Estos sustratos presentan raíces con una estructura muy ramificada y en la mayoría de los casos flotantes en la columna de agua, lo cual puede facilitar la colonización y adherencia de algas con o sin estructuras de fijación. Osorio y Manjarres (2015) registraron para Cerro de San Antonio (norte de Colombia), un comportamiento similar, donde E. crassipes arrojó los mayores valores de densidad ficoperifítica, relacionando la arquitectura de la raíz con la densidad algal. Gomphonema, Chroococcus y Cosmarium fueron comunes para todas las macrófitas analizadas en esta estación de muestreo (Figura 2). Algunas de las especies de estos géneros, especialmente de Chroococcus y Cosmarium, son de hábitat planctónico comunes en aguas mesotróficas y oligotróficas (Ramírez, 2000) por lo que su presencia en las raíces pudo deberse a la mezcla frecuente del agua por la acción del viento dado lo somero del sistema (Montoya-Moreno y Aguirre, 2013b).

En Caño Venado (E2) se registraron 1809 células distribuidas en 5 clases, 21 familias y 27 géneros. Bacillariophyceae fue la clase más abundante (47\%) registrando una densidad de 854 células $/ \mathrm{cm}^{2}$, seguida de Chlorophyceae con una densidad de 578 células $/ \mathrm{cm}^{2}$ equivalentes al $32 \%$. De igual forma, fueron registradas las clases Cyanophyceae con un 13 \% (222 células / $\mathrm{cm}^{2}$ ), Conjugatophyceae con $8 \%$ (150 células $/ \mathrm{cm}^{2}$ ) y Euglenophyceae con 0,2 \% (5 células $/ \mathrm{cm}^{2}$ ). Esta estación a pesar de presentar mayor riqueza de macrófitas, evidencio baja diversidad algal, lo cual pudo relacionarse con factores como la herbivoría, la competencia con el fitoplancton y la alta carga de sedimento, pues son considerados como los principales factores bióticos que regulan el crecimiento ficoperifítico (Oliveira-Martins, 2006). 


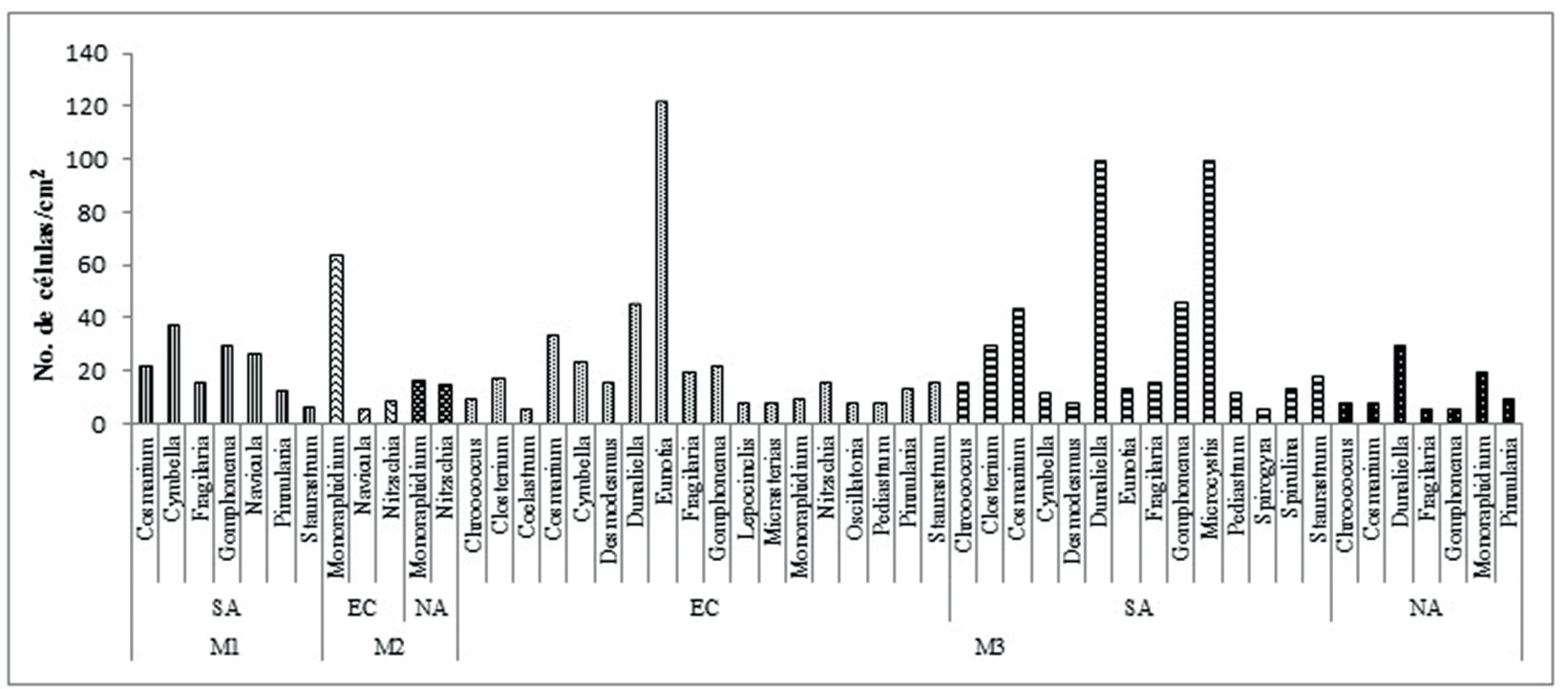

Figura 2. Géneros ficoperifíticos más representativos con valores mayores a 5 células / $\mathrm{cm}^{2}$ presentes en las macrófitas evaluadas en la estación Rincón del Burro (E1). SA = Salvinia auriculata, $\mathrm{EC}=$ Eichhornia crassipes, NA = Najas arguta . M1 = junio de 2015, M2 = septiembre de 2015, M3 = enero de 2016.

Los géneros dominantes en junio de 2015 fueron Chroococcus y Dunaliella para S. auriculata y Cymbella y Fragilaria para E. crassipes (Figura 3). En enero de 2016, el género dominante fue Dunaliella alcanzando las mayores abundancias en los sustratos E. crassipes, S. auriculata y Mimosa sp., mientras que para Marsilea sp. y $P$. repens el género dominante fue Monoraphidium (Figura 3).

La presencia de Chroococcus (Cianofita) en todas las macrófitas examinadas en Caño Venado, indicó estratificación de aguas y empobrecimiento por contaminación, ya que se halla invariablemente en hábitats donde el agua es más acida (Márquez y Guillot, 1988). De La Parra (2015) registró el mismo género en un sector de la cuenca baja del Río Cesar (norte de Colombia) donde desembocan aguas residuales.

Para Botilleros (E3), en septiembre de 2015, se registraron un total de 809 células, correspondientes a 4 clases, 15 familias y 20 géneros de las cuales el $40 \%$ (324 células $/ \mathrm{cm}^{2}$ ) fueron de la clase Chlorophyceae, seguida de Bacillariophyceae con el 35 \% (280 células $\left(\mathrm{cm}^{2}\right)$. De igual forma, la clase Conjugatophyceae registró una densidad de 177 células / $\mathrm{cm}^{2}$ equivalente al $22 \%$ y Cyanophyceae significó un $3 \%$ registro 28 células $/ \mathrm{cm}^{2}$. Los géneros Dunaliella, Cosmarium y Gomphonema, además de ser comunes en todos los sustratos evaluados, registraron la mayor abundancia para esta estación (Figura 4). Al igual que en Rincón del Burro, se encontraron géneros de algas verdes (Clorofíceas) de hábito planctónico (Cosmarium, Spirogyra, Pediastrum, Desmodesmus) comunes en aguas mesotróficas y oligotróficas (Ramírez, 2000). Se registró el género Closterium, cuyas especies son consideradas cosmopolitas, por lo general presentes en aguas ácidas, rara vez en aguas alcalinas que, cuando su densidad es alta, pueden conferirle olor a pepino al agua (Ramírez, 2000).

En general, las diatomeas predominan en este tipo de ambientes debido a su rapidez para ocupar sustratos en corto tiempo, además, presentan estructuras especializadas que les permiten fijarse al sustrato con mayor facilidad y a su vez competir con ventaja sobre otras especies. Se presentaron altas densidades de clorófitas también, indicando una alta carga orgánica y una penetración lumínica permanente que favorece su crecimiento (Montoya-Moreno y Aguirre, 2013b). La macrófita $E$. crassipes fue el sustrato más colonizado por las algas del perifíton, exceptuando la estación de Botilleros, en la cual se presentó una alta carga de material orgánico e inorgánico. El sustrato E. crassipes presenta raíces muy ramificadas y flotantes en la columna de agua, lo cual permite a las algas perifíticas colonizar y adherirse fácilmente. 


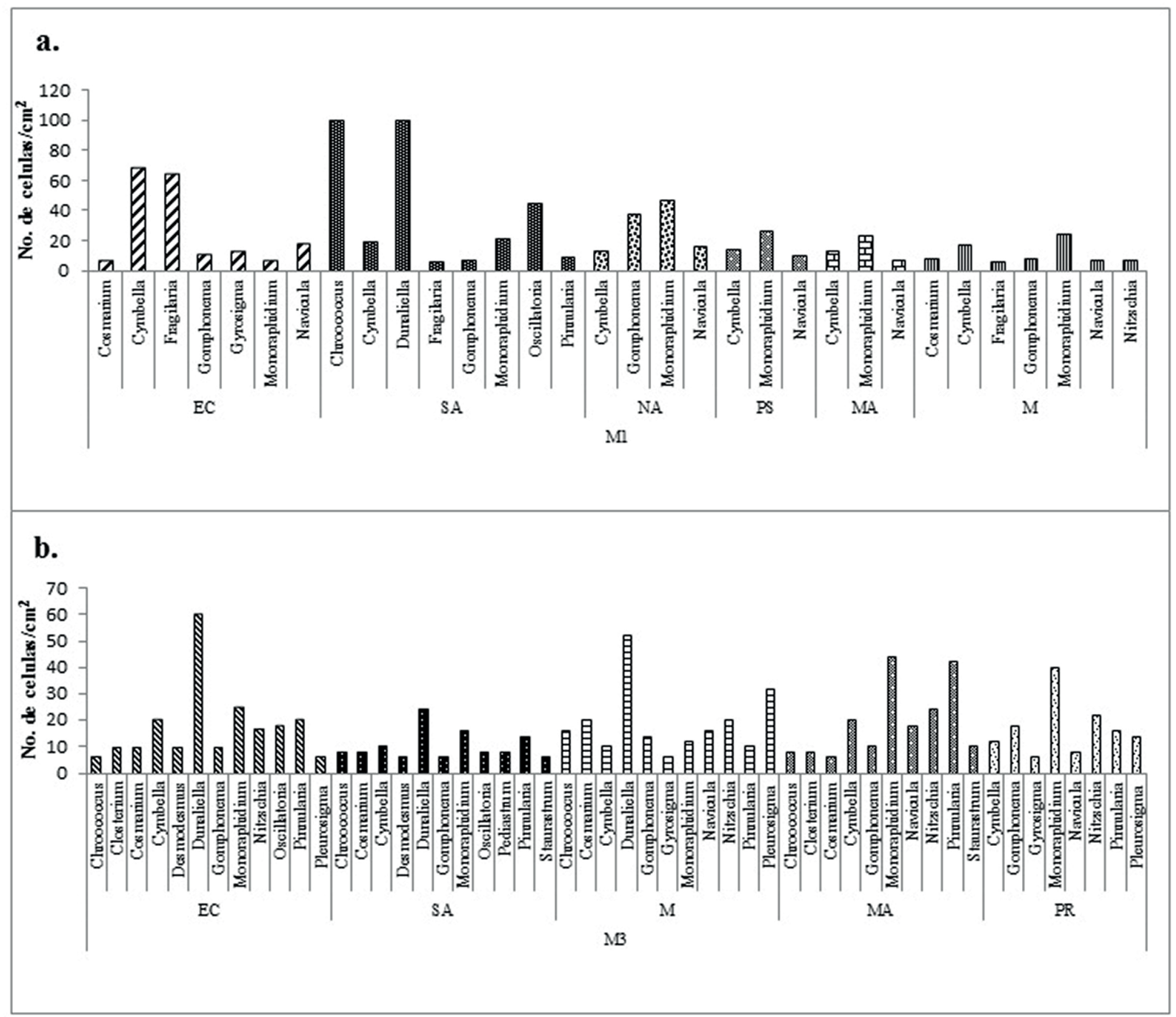

Figura 3. Géneros ficoperifíticos más representativas con valores mayores a 5 células / $\mathrm{cm}^{2}$ de las macrófitas evaluadas en la estación Caño Venado (E2). EC = Eichhornia crassipes, $\mathrm{SA}=$ Salvinia auriculata, $\mathrm{NA}=$ Najas arguta, $\mathrm{PS}=$ Pistia stratiotes, $\mathrm{MA}=\mathrm{Marsilea}$ sp., $\mathrm{M}=$ Mimosa sp., PR = Paspalum repens. M1 = junio de 2015, M3 = enero de 2016. 


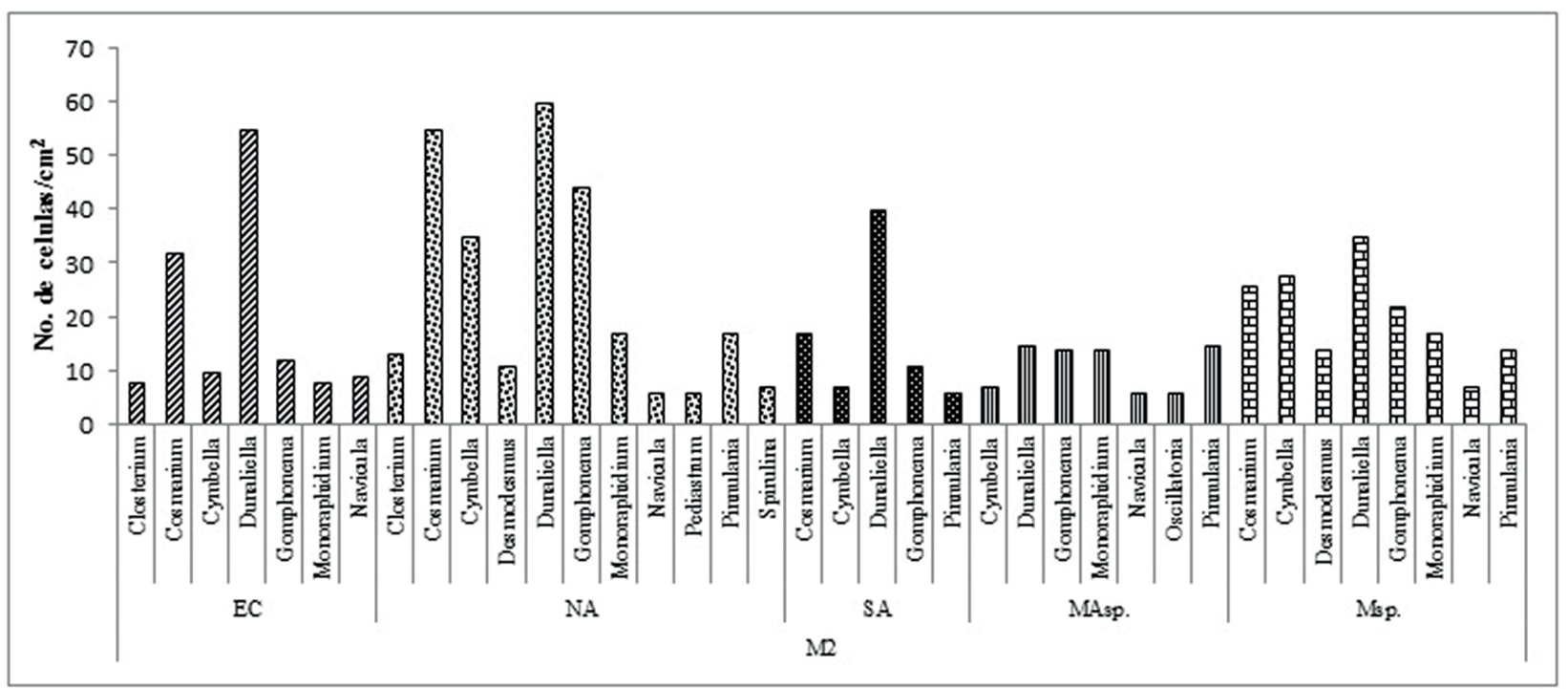

Figura 4. Géneros ficoperifíticos más representativos con valores mayores a 5 células / $\mathrm{cm}^{2}$ de las macrófitas evaluadas en la estación Botilleros (E3) $. \mathrm{EC}=$ Eichhornia crassipes, $\mathrm{SA}=$ Salvinia auriculata, $\mathrm{NA}=$ Najas arguta, $\mathrm{MA}=$ Marsilea $\mathrm{sp} ., \mathrm{M}=$ Mimosa sp. $\mathrm{M} 2=$ septiembre de 2015.

El presente estudio se constituye como una línea base para el monitoreo de la ciénaga de Chilloa, ya que las algas perifíticas por su alta tasa de reciclaje de nutrientes han de desempeñar un papel fundamental en la dinámica de este humedal, además de que ellas son consideradas como buenas indicadoras de la calidad de agua.

\section{AGRADECIMIENTOS}

Este estudio fue financiado por la Fundación para la Participación; Capacitación y la Investigación Social "FUPARCIS" y la Gobernación del Magdalena, en el marco del proyecto INVESTIGACIÓN PARA LA CARACTERIZACIÓN, ZONIFICACIÓN, ORDENAMIENTO, RESTAURACIÓN Y MANEJO DE LOS HUMEDALES DEL DEPARTAMENTO DEL MAGDALENA, CARIBE. Código BPIN: 2013000100017.

\section{BIBLIOGRAFIA}

Algarte, V.M. 2014. O efeito dos fatores locais e de preditores espaciais sobre a estrutura das algas perifíticas em planície de inundação: uma perspectiva de metacomunidade. Tese de doutoramento, Universidade Estadual de Maringá, Maringá, Brazil.

Bicudo, E.M. y Menezes, M. 2006. Gêneros de algas de águas continentais. do Brasil (Chave de identificação e descrições). 2 Ed. RiMa Editora, São Carlos, Brasil.
Bourrelly, P. 1970. Les algues d'eau douce. N. Boubée y Cie, Paris.

Bourrelly, P. 1972. Les algues d'eau douce. N. Boubée y Cie, Paris.

Bourrelly, P. 1981. Les algues d'eau douce. N. Boubée y Cie, Paris.

Comité Européen de Normalisation "CEN". 2003. Water quality-guidance standard for the routine sampling and pretreatment of benthic diatoms from rivers. British Standart, Brussels.

De La Parra, A. 2015. Composición y estructura del fitoperifiton y Metabolismo de la Cuenca Baja del Río Cesar, Cesar - Colombia. Cesar, Colombia. Tesis de maestría, Universidad del Atlántico, Barranquilla, Colombia.

Díaz-Quirós, C. y Rivera-Rondón, C. 2004. Diatomeas de pequeños ríos andinos y su utilización como indicadoras de condiciones ambientales. Caldasia 26(2): 381-394.

Donato, J., González, L. y Rodríguez, C. 1996. Ecología de dos sistemas acuáticos de páramo. Academia Colombiana de Ciencias Exactas, Físicas y Naturales, Bogotá, Colombia.

Fauth, J., Bernardo, J., Camara, M., Resetarits, W., Van Buskirk, J. y McCollum, S. 1996. Simplifying the jargon of community ecology: a conceptual approach. American naturalist 147(2): 282-286. 
Fundación para la Participación, Capacitación y la Investigación Social "FUPARCIS". 2014. Información Proyecto Humedales del Magdalena._URL:_http://www. fuparcis.org/default/proyectoHumedales.php?current = proyectosyidContent $=7$. Consultado: 17 de Abril 2015 .

Goldsborough, L., McDougal, R. y North, A. 2005. Periphyton in Freshwater Lakes and Wetlands. En: Azim, M.E., Verdegem, M.C., Van Dam, A.A. y Beveridge, M.C., Editores. Periphyton: Ecology, exploitation and management. CABI Publishing, Cambridge.

Guiry, M.D. 2015. AlgaeBase. World-wide electronic publication, National University of Ireland, Galway._URL:_ http://www.algaebase.org. Consultado: 13 de febrero 2016.

Hauer, F.R. y Lamberti, G.A. 2007. Methods in stream ecology. Academic Press, San Diego.

Hernández-Atilano, E., Aguirre, J. y Palacio, J. 2005. Variación espacio-temporal de la estructura de la comunidad de algas perifíticas en la microcuenca de la Quebrada la Vega, municipio de San Roque (Antioquia), Colombia. Actualidades Biológicas 27(82): 67-77.

Kiss, M., Lakatos, G., Borics, G., Gido, Z. y Deak, C. 2003. Littoral macrophyte-periphyton complexes in two Hungarian shallow waters. Hydrobiologia 506(1): 541-548.

Komárek, J. y Anagnostidis, K. 1999. Cyanoprokaryota, Part 1: Chroococcales. En: Ettl, H., Gerloff, J., Heynig, H. y Mollenhauer, D., Editores. Süsswasserflora von Mitteleuropa Bd 19/1. Gustav Fischer Verlag, Stuttgart.

Komárek, J. y Anagnostidis, K. 2005. Cyanoprokaryota, Part 2: Oscillatoriales. En: Büdel, B., Gärtner, G., Krienitz, L. y Schagerl, M., Editores. Süsswasserflora von Mitteleuropa Bd 19/2. Spektrum Akademischer Verlag, Heidelberg.

Krammer, K. y Lange-Bertalot, H. 1986. Bacillariophyceae, Part 1: Naviculaceae. En: Ettl, H., Gerloff, J., Heynig, H. y Mollenhauer, D., Editores. Susswasserflora von Mittleuropa Bd 2/1. Gustav Fisher Verlag, Stuttgart.

Krammer, K. y Lange-Bertalot, H. 1988. Bacillariophyceae, Part 2: Bacillariaceae, Epithemiaeeae, Surirellaceae. En: Ettl, H., Gerloff, J., Heyning, H. y Mollenhauer, D., Editores. Susswasserflora von Mittleuropa Bd 2/2. Gustav Fischer 134 Verlag, Stuttgart.

Krammer, K. y Lange-Bertalot, H. 1991. Bacillarophyceae, Part 3: Centrales, Fragilariaceae, Eunotiaceae. En: Ettl, H., Gerloff, J., Heyning, H. y Mollenhauer, D., Editores.
Süßwasserflora von Mitteleuropa Bd 2/3. Gustav Fischer Verlag, Sttugart.

Lange-Bertalot, H. 2001. Navicula sensu stricto 10 genera separated from Navicula sensu lato Frustulia. En: LangeBertalot, H., Editor. Diatoms of Europe. Gantner Verlag, Ruggell.

Márquez, G. y Guillot, G. 1988. Proyecto estudios ecológicos de embalses colombianos. Etapa prospectiva. Informe final, FEN-Universidad Nacional de Colombia, Bogotá.

Montoya-Moreno, Y. y Ramírez, J. 2007. Variación estructural de la comunidad perifítica colonizadora de sustratos artificiales en la zona de Ritral del río Medellín, Colombia. Revista de Biología Tropical 55(2): 585-593.

Montoya-Moreno, Y. y Aguirre, N. 2008. Asociación de algas perifíticas en raíces de macrófitas en una ciénaga tropical Colombiana. Hidrobiologia 18(3): 189-198.

Montoya-Moreno, Y. y Aguirre, N. 2009. Estado del arte de la limnología de lagos de planos inundables (ciénagas) en Colombia. Revista Gestión y Ambiente 12(3): 85-106.

Montoya-Moreno, Y. y Aguirre, N. 2013a. Estado de arte del conocimiento sobre perifíton en Colombia. Revista Gestión y Ambiente 16(3): 91-117.

Montoya-Moreno, Y. y Aguirre, N. 2013b. Dinámica del ensamblaje algal epifítico en el sistema de planos inundables de Ayapel a través del pulso de inundación. Revista U.D.C.A Actualidad y Divulgación Científica 16(2): 491-500.

Oliveira-Martins, F. 2006. Respostas ecofisiologicas da comunidade perifitica in situ a diferentes condicoes ambientais no Rio Santa da Vitoria. Tesis de doctorado, Universidade Federal Do Espiritu Santo, Vitoria, Brasil.

Osorio, F. y Manjarres, G. 2015. Ficoperifíton asociado a macrófitas en la ciénaga Cerro de San Antonio, Magdalena - Colombia. Intropica 10: 74-83.

Ramírez, J. 2000. Fitoplancton de agua dulce: aspectos ecológicos, taxonómicos y sanitarios. Universidad de Antioquia, Medellín, Colombia.

Ramírez, A. y Gutiérrez-Fonseca, P. 2016. Sobre ensambles y ensamblajes ecológicos - respuesta a Monge - Nájera. Revista de biología tropical 64(2): 817-819. 
Sand-Jensen, K. 1983. Physical and chemical parameters regulating growth of Periphytic communities. En: Wetzel, R., Editor. Periphyton of Freshwater Ecosystems. Springer Science and Business Media, Boston.

Sierra, O. y Ramírez, J. 2000. Variación espacio-temporal de biopelículas en la represa La Fe, el Retiro, Antioquia (Colombia). Actualidades Biológicas 22(73): 153-168.

Stroud, J., Bush, M., Ladd, M., Nowicki, R., Shantz, A. y Sweatman, J. 2015. Is a community still a community? Reviewing definitions of key terms in community ecology. Ecology and Evolution 5(21): 4757-4765.
Vadeboncoeur, Y. y Steinman, A. 2002. Periphyton Function in Lake Ecosystems. The Scientific World Journal 2: 1449-1468.

Velásquez, J. 1994. Plantas acuáticas vasculares de Venezuela. Universidad Central de Venezuela, Caracas, Venezuela.

Wetzel, R. 2001. Limnology: Lake and River Ecosystems. Academic Press, San Diego.

Fecha de recepción: 30/06/2016

Fecha de aceptación: 01/12/2016

Para citar este artículo: De La Hoz-Barrientos, L.A. y Osorio-Ávila, F.J. 2016. Ensamble ficoperifitíco asociado a macrófitas en una ciénaga tropical colombiana.

Revista Intropica Vol. 11: 127 - 135 